\title{
STABILITY RESULTS FOR FIRST ORDER PROJECTION BODIES
}

\author{
P. R. GOODEY AND H. GROEMER
}

(Communicated by William J. Davis)

\begin{abstract}
The motivation for this work comes from a result of Minkowski. He showed that if a three-dimensional convex body has the property that all its projections have the same perimeter, then the original body has constant width. Our objective was to extend this to a stability result and not to restrict ourselves to dimension three. The result we obtained shows that if two centrally symmetric bodies have projections which all have approximately the same mean width, then the two bodies are approximately the same up to translation. This is, in effect, a continuity result for the inverse of the spherical Radon transform. It is closely related to recent three-dimensional results of Campi and to work of Bourgain and Lindenstrauss, who consider the volumes of projections rather than their mean widths. The techniques we employ are drawn from the theory of spherical harmonics and from the theory of mixed volumes.
\end{abstract}

\section{INTRODUCTION}

The investigation we are presenting here has its origin in the work of Minkowski [11]. We let $K$ be a convex body in three-dimensional Euclidean space. If $K$ is of constant width it is easily seen that the perimeter of the orthogonal projection of $K$ onto any plane is constant (that is, independent of the plane). Using spherical harmonics, Minkowski proved the converse of this theorem: If the perimeter of these projections is constant then $K$ is of constant width. This result has been generalized in various directions. For example, it is possible to remove the restriction that $K$ is three-dimensional (see Schneider [15]) and the theorem can be stated within the more general framework of projection bodies (this will be explained in the next section). Another interesting variation of Minkowski's theorem concerns the associated stability problem: How much does $K$ deviate from a convex body of constant width if the perimeter of the projections is, in some sense, approximately constant? For three-dimensional bodies this problem has been investigated by Campi [5]. More recently Bourgain and Lindenstrauss [4] have solved the related stability problem regarding areas of projections (see also Campi [5] for the three-dimensional case).

Received by the editors May 20, 1989.

1980 Mathematics Subject Classification (1985 Revision). Primary 52A20; Secondary 52A40.

The second author was supported by National Science Foundation Research Grant DMS8701893. 
The aim of the present article is to prove stability theorems for convex bodies of arbitrary dimension and to do this in a simple, direct, and transparent manner. Although we will follow Campi (and indeed Minkowski) in using spherical harmonics, our approach to the problem is somewhat different.

\section{THE MAIN THEOREM}

Let $\mathbb{E}^{d}$ denote Euclidean $d$-dimensional space $(d \geq 3)$. The $(d-1)$ dimensional unit sphere in $\mathbb{E}^{d}$ will be denoted by $S^{d-1}$, and if $u \in S^{d-1}$ we let $E(u)$ denote the $(d-1)$-dimensional linear subspace that is orthogonal to $u$. If $K$ is a compact body in $\mathbb{E}^{d}$ (that is, a compact convex subset of $\mathbb{E}^{d}$ with interior points), we let $K_{u}$ denote the orthogonal projection of $K$ onto $E(u)$. Thus, $K_{u}$ is a $(d-1)$-dimensional convex body. As usual, $W_{k}$ denotes the $k$ th Quermassintegral in $\mathbb{E}^{d}$ and $W_{k}^{\prime}$ the corresponding Quermassintegral in $\mathbb{E}^{d-1}$. In particular $W_{d}(K)=\kappa_{d}=\omega_{d} / d$, where $\kappa_{d}$ denotes the volume and $\omega_{d}$ the surface area of the unit ball in $\mathbb{E}^{d}$. We also introduce the mean width of $K$, which can be defined by

$$
M(K)=\frac{2}{\kappa_{d}} W_{d-1}(K) \text { or by } M(K)=\frac{2}{\omega_{d}} \int_{S^{d-1}} H_{K}(u) d \sigma(u),
$$

where $H_{K}$ denotes the support function of $K$ and $\sigma$ the surface area measure on $S^{d-1}$.

If $K$ and $L$ are two convex sets in $\mathbb{E}^{d}$ with respective support functions $H_{K}$ and $H_{L}$, we introduce as an indicator of the deviation of $K$ from $L$ (or $L$ from $K$ ) the $L_{2}$-distance

$$
h_{2}(K, L)=\left(\int_{S^{d-1}}\left(H_{K}(u)-H_{L}(u)\right)^{2} d \sigma(u)\right)^{1 / 2} .
$$

For the investigation of stability questions it is often more convenient to use $h_{2}(K, L)$ instead of the more familiar Hausdorff (or supremum norm) distance $h(K, L)$ (cf. Campi [5], Groemer [7], and Schneider [17]). The relationship between $h_{2}$ and $h$ has been studied by Vitale [19]. In particular, Vitale has shown that

$$
\alpha_{1} h_{2}(K, L) \leq h(K, L) \leq \alpha_{2} D^{2(d-1) /(d+1)} h_{2}(K, L)^{2 /(d+1)},
$$

with

$$
\alpha_{1}=1 / \sqrt{\omega_{d}}, \quad \alpha_{2}=\left(B\left(\frac{1}{2}, \frac{d-1}{2}\right)\right) /\left(\sqrt{\omega_{d}} B(3, d-1)\right)^{2 /(d+1)},
$$

where $D=\operatorname{diam}(K \cup L)$ and $B$ is the beta-function. These inequalities would enable us to formulate all our results in terms of $h$ instead of $h_{2}$, but in general we will not do this.

Let us now assume that both $K$ and $L$ are centrally symmetric about the origin $o$ of $\mathbb{E}^{d}$. Our problem is to estimate $h_{2}(K, L)$ (or $h(K, L)$ ) under the assumption that for every $u \in S^{d-1}$ the expression

$$
\left|W_{d-2}^{\prime}\left(K_{u}\right)-W_{d-2}^{\prime}\left(L_{u}\right)\right|
$$


or equivalently

$$
\left|M\left(K_{u}\right)-M\left(L_{u}\right)\right|,
$$

is small. Actually, it will again be more convenient (and weaken our assumptions) if we use the $L_{2}$-norm $\|\cdot\|_{2}$ on $C\left(S^{d-1}\right)$ rather than the supremum norm. Our principal result is the following theorem.

Theorem 1. Let $K$ and $L$ be two convex bodies in $\mathbb{E}^{d}$ which are centrally symmetric with respect to the origin. If for some $\varepsilon \geq 0$

$$
\left\|M\left(K_{u}\right)-M\left(L_{u}\right)\right\|_{2} \leq \varepsilon
$$

then

$$
h_{2}(K, L) \leq \varepsilon^{2 / d} \frac{1}{2}\left(\lambda_{d}(\delta(K)+\delta(L))+\varepsilon^{2}\right)^{(d-2) / 2 d},
$$

where

$$
\delta(\cdot)=\frac{1}{\kappa_{d}} W_{d-1}^{2}(\cdot)-W_{d-2}(\cdot)
$$

and

$$
\lambda_{d}=8 d(d-1)^{2} / \mu_{d}^{4 /(d-2)}
$$

with $\mu_{3}=1 / \sqrt{2}$ and

$$
\mu_{d}=\begin{aligned}
& 1.3 \ldots(d-3) \text { if } d \text { is even } \\
& 2.4 \ldots(d-3) / \sqrt{2} \text { if } d \text { is odd }
\end{aligned}
$$

It is known that $\delta(K) \geq 0$ for every convex body $K$ in $\mathbb{E}^{d}$, with equality if and only if $K$ is a ball. Further remarks regarding $\delta$ can be found in $\S 6$.

We mention another interpretation of Theorem 1. It is known (see Bonnesen and Fenchel [3]) that $W_{d-j-1}^{\prime}\left(K_{u}\right)$, considered as a function of $u$, is the support function of a centrally symmetric convex body in $\mathbb{E}^{d}$. This convex body is known as the $j$ th order projection body of $K$ and is denoted by $\Pi_{j} K$. Using this notation, condition (2) can then be written in the form

$$
h_{2}\left(\Pi_{1} K, \Pi_{1} L\right) \leq \frac{\kappa_{d}}{2} \varepsilon,
$$

and Theorem 1 may be interpreted as a stability result for the function $\Pi_{1}^{-1}$. Further information regarding the stability of the $\Pi_{j}$ and $\Pi_{j}^{-1}$ for $1 \leq j \leq d-1$ can be found in Schneider and Weil [18] and Goodey [6].

The proof of Theorem 1 will be given in $\S 4$. First we have to establish several auxiliary results.

\section{THREE LEMMAS}

In this section we use techniques from the theory of spherical harmonics to establish some inequalities which will form the basis of our main result. Standard references for spherical harmonics include Müller [12] and Helgason [8]. 
If $f$ is a continuous function on $S^{d-1}$, then we can associate with $f$ the series

$$
\sum_{n=0}^{\infty} Q_{n}
$$

where the terms $Q_{n}$ are spherical harmonics of order $n$ which are determined in the usual way. We recall that spherical harmonics of degree $n$ are eigenvalues of the Laplace-Beltrami operator $\Delta$ on $S^{d-1}$. In fact, if $u$ is such a function then

$$
\Delta u=-n(n+d-2) u
$$

(see for example, Berg [2], Helgason [8], or Müller [12]). We denote the relationship between $f$ and its associated series by

$$
f \sim \sum_{n=0}^{\infty} Q_{n}
$$

and recall that Parseval's equation shows that

$$
\|f\|_{2}^{2}=\sum_{n=0}^{\infty}\left\|Q_{n}\right\|_{2}^{2} \text {. }
$$

More generally, if $g$ and $h$ are continuous functions on $S^{d-1}$ with

$$
g \sim \sum_{n=0}^{\infty} a_{n} Q_{n} \text { and } h \sim \sum_{n=0}^{\infty} b_{n} Q_{n}
$$

then

$$
\int_{S^{d-1}} g(u) h(u) d \sigma(u)=\sum_{n=0}^{\infty} a_{n} b_{n}\left\|Q_{n}\right\|_{2}^{2},
$$

where the latter series converges absolutely. These standard results will be used repeatedly without specific reference.

If $p \in S^{d-1}$ we let $S_{p}$ denote the $(d-2)$-dimensional sphere $S^{d-1} \cap E(p)$. The surface area measure on $S_{p}$ will be denoted by $\sigma_{p}$. We let $C_{n}^{\nu}(x)$ denote the Gegenbauer polynomials; they can be defined by

$$
\left(1-2 x \xi+\xi^{2}\right)^{-\nu}=\sum_{m=0}^{\infty} C_{n}^{\nu}(x) \xi^{n} .
$$

In this section it is always assumed that $K$ and $L$ are two convex bodies in $\mathbb{E}^{d}$ that are centrally symmetric with respect to $o$. Furthermore, we set

$$
H=H_{K}-H_{L}
$$

and write

$$
H \sim \sum^{*} X_{n}
$$

where $\sum^{*}$ is an abbreviation $\sum_{n=0}^{\infty}(n$ even $)$; note that $X_{n}=0$ if $n$ is odd. 
Lemma 1. If we set

$$
M(H, p)=M\left(K_{p}\right)-M\left(L_{p}\right)=\frac{2}{\omega_{d-1}} \int_{S_{p}} H d \sigma_{p},
$$

then

$$
\|M(H, P)\|_{2}^{2}=4 \sum^{*} \gamma_{n}^{2}\left\|X_{n}\right\|_{2}^{2},
$$

where

$$
\gamma_{n}=C_{n}^{\nu}(0) / C_{n}^{\nu}(1) \quad \text { with } \quad \nu=\frac{d-2}{2} \text {. }
$$

Proof. Schneider $[14,15]$ has shown by direct calculation that

$$
\int_{S_{p}} X_{n} d \sigma_{p}=\omega_{d-1} \gamma_{n} X_{n}(p)
$$

As he points out, this can also be deduced from the Funk-Hecke theorem; it is also a consequence of the intertwining property of the Laplace-Beltrami operator with the Radon transform (see Helgason [8]). If we set $t_{k}=1-(1 / k)$ $(k=1,2, \ldots)$ and

$$
H^{k}=H^{k}(u)=\sum^{*} t_{k}^{n} X_{n}(u),
$$

then this series converges uniformly in $u$ for every $k$, see Müller [12]. Furthermore,

$$
\lim _{k \rightarrow \infty} H^{k}(u)=H(u)
$$

uniformly in $u$. It follows immediately that

$$
M(H, p)=\lim _{k \rightarrow \infty} M\left(H^{k}, p\right) .
$$

Using this and (4) we find

$$
\begin{aligned}
\|M(H, p)\|_{2}^{2} & =\lim _{k \rightarrow \infty}\left\|M\left(H^{k}, p\right)\right\|_{2}^{2}=\lim _{k \rightarrow \infty} 4\left\|\sum^{*} \gamma_{n} t_{k}^{n} X_{n}\right\|_{2}^{2} \\
& =\lim _{k \rightarrow \infty} 4 \sum^{*} \gamma_{n}^{2} t_{k}^{2 n}\left\|X_{n}\right\|_{2}^{2} .
\end{aligned}
$$

Since $t_{k} \leq 1, t_{k} \rightarrow 1$ this implies

$$
\begin{aligned}
4 \sum_{n \leq N}^{*} \gamma_{n}^{2}\left\|X_{n}\right\|_{2}^{2} & =\lim _{k \rightarrow \infty} 4 \sum_{n \leq N}{ }^{*} \gamma_{n}^{2} t_{k}^{2 n}\left\|X_{n}\right\|_{2}^{2} \\
& \leq\|M(H, p)\|_{2}^{2} \leq 4 \sum^{*} \gamma_{n}^{2}\left\|X_{n}\right\|_{2}^{2}
\end{aligned}
$$

and the lemma follows by letting $N \rightarrow \infty$.

Lemma 2. If $\gamma_{n}$ is defined as in Lemma 1 and $n=2,4, \ldots$, then

$$
1 / \gamma_{n}^{2} \leq((d-1)(n-1)(n+d-1))^{(d-2) / 2} / \mu_{d}^{2},
$$

with $\mu_{d}$ as in Theorem 1 .

Proof. The definition of $C_{n}^{\nu}(x)$ shows that $C_{n}^{\nu}(0)=\left(\begin{array}{c}-\nu \\ n / 2\end{array}\right)$ and $C_{n}^{\nu}(1)=\left(\begin{array}{c}-2 \nu \\ n\end{array}\right)$. Hence (remembering that $n$ is even), we find

$$
\frac{1}{\gamma_{n}}=\frac{C_{n}^{\nu}(1)}{C_{n}^{\nu}(0)}=(-1)^{n / 2} \frac{(d-1)(d+1) \cdots(d+n-3)}{1.3 \ldots(n-1)} \text {. }
$$


Essentially the same calculation can be found in Schneider [13]. If $d$ is even it follows that

$$
\left|\frac{1}{\gamma_{n}}\right|=\frac{1}{\mu_{d}}(n+1)(n+3) \cdots(d+n-3) .
$$

Consequently,

$$
1 / \gamma_{n}^{2} \leq \frac{1}{\mu_{d}^{2}}(n+d-3)^{(d-2) / 2}(n+d-1)^{(d-2) / 2},
$$

and the lemma follows since for $d \geq 3, n \geq 2$ we have

$$
n+d-3 \leq(n-1)(d-1) \text {. }
$$

To prove our statement when $d$ is odd, we first note that an obvious induction argument implies

$$
\frac{2.4 \ldots n}{1.3 \ldots(n-1)} \leq \sqrt{2 n} \text {. }
$$

Now we have

$$
\sqrt{2}\left|\frac{1}{\gamma_{n}}\right|=\frac{1}{\mu_{d}} \frac{2.4 \ldots n}{1.3 \ldots(n-1)}(n+2) \cdots(n+d-3) .
$$

Hence,

$$
\begin{aligned}
1 / \gamma_{n}^{2} & \leq \frac{1}{\mu_{d}^{2}} n((n+2) \cdots(n+d-3))^{2} \\
& \leq \frac{1}{\mu_{d}^{2}}(n+d-3)^{d-2} \\
& \leq \frac{1}{\mu_{d}^{2}}(n+d-3)^{(d-2) / 2}(n+d-1)^{(d-2) / 2},
\end{aligned}
$$

and the lemma follows as before.

Before continuing, we briefly recall some of the results of Berg [2] which extend the notion of spherical harmonic expansions to the setting of distributions. He explains that the convolution of a function in $C^{\infty}\left(S^{d-1}\right)$ with a distribution on $S^{d-1}$ is again a function in $C^{\infty}\left(S^{d-1}\right)$. Furthermore the convolution of the Legendre polynomial of degree $n$ with a distribution gives a spherical harmonic of degree $n$. These convolutions (or certain multiples of them) provide the entries in the spherical harmonic expansion of the distribution. So it makes sense to write

$$
T \sim \sum_{n=0}^{\infty} S_{n}
$$

in the case where $T$ is a distribution. Most importantly, Berg shows that this is consistent with the embedding of $C\left(S^{d-1}\right)$ in the space of distributions on $S^{d-1}$. These observations, together with (3), show that if $G$ is a continuous function on $S^{d-1}$ with

$$
G \sim \sum_{n=0}^{\infty} S_{n}
$$


then $\Delta G$ is a distribution with spherical harmonic expansion given by

$$
\Delta G \sim-\sum_{n=0}^{\infty} n(n+d-2) S_{n} .
$$

Lemma 3. Let $G$ be the support function of a convex body $K$ in $\mathbb{E}^{d}$ and assume that $G \sim \sum_{m=0}^{\infty} U_{m}$. Then

$$
\sum_{m=2}^{\infty}(m-1)(m+d-1)\left\|U_{m}\right\|_{2}^{2}=d(d-1) \delta(K) .
$$

Proof. It follows from (5) that

$$
\int_{S^{d-1}} G\left(\frac{1}{d-1} \Delta G+G\right) d \sigma=-\frac{1}{d-1} \sum_{m=0}^{\infty}(m-1)(m+d-1)\left\|U_{m}\right\|_{2}^{2} .
$$

Next we recall from Bonnesen and Fenchel [3] or Berg [2] that if $K$ is sufficiently smooth, then

$$
W_{d-2}(K)=\frac{1}{d} \int_{S^{d-1}} G\left(\frac{1}{d-1} \Delta G+G\right) d \sigma .
$$

Of course, even if $K$ is not smooth this result holds, but $\Delta G$ is now a distribution. Combining this with the fact that

$$
U_{0}=\frac{\sqrt{\omega_{d}} M(K)}{2}=\sqrt{\frac{d}{\kappa_{d}}} W_{d-1}(K),
$$

we obtain the required result

$$
\begin{aligned}
\sum_{m=2}^{\infty} & (m-1)(m+d-1)\left\|U_{m}\right\|_{2}^{2} \\
& =-d(d-1) W_{d-2}(K)+(d-1) \frac{d}{\kappa_{d}} W_{d-1}^{2}(K) \\
& =d(d-1) \delta(K) .
\end{aligned}
$$

For a somewhat different approach to relations of this kind see Schneider [17] and Kubota [9]. The use of distributions in this proof is convenient, but not essential. It would suffice to establish our theorems only for sufficiently smooth bodies. The general case can then be obtained by standard approximation arguments.

\section{Proof of Theorem 1}

Since (2) is equivalent to $\|M(H, p)\|_{2} \leq \varepsilon$, we obtain from Lemma 1

$$
\sum^{*} \gamma_{n}^{2}\left\|X_{n}\right\|_{2}^{2} \leq \frac{1}{4} \varepsilon^{2}
$$


Hence, using Hölder's inequality and Lemma 2 we find

$$
\begin{aligned}
\sum^{*}\left\|X_{n}\right\|_{2}^{2} & =\sum^{*}\left(\gamma_{n}^{4 / d}\left\|X_{n}\right\|_{2}^{4 / d}\right)\left(\gamma_{n}^{-4 / d}\left\|X_{n}\right\|_{2}^{2-(4 / d)}\right) \\
& \leq\left(\sum^{*} \gamma_{n}^{2}\left\|X_{n}\right\|_{2}^{2}\right)^{2 / d}\left(\left\|X_{0}\right\|_{2}^{2}+\sum_{n \geq 2}^{*} \gamma_{n}^{-4 /(d-2)}\left\|X_{n}\right\|_{2}^{2}\right)^{(d-2) / d} \\
& \leq\left(\frac{\varepsilon}{2}\right)^{4 / d}\left(\frac{(d-1)}{\mu_{d}^{4 /(d-2)}} \sum_{n \geq 2}^{*}(n-1)(n+d-1)\left\|X_{n}\right\|_{2}^{2}+\left\|X_{0}\right\|_{2}^{2}\right)^{(d-2) / d} .
\end{aligned}
$$

If we now assume that $H_{K} \sim \sum^{*} Y_{n}$ and $H_{L} \sim \sum^{*} Z_{n}$, it follows that

$$
\left\|X_{n}\right\|_{2}^{2}=\left\|Y_{n}-Z_{n}\right\|_{2}^{2} \leq\left(\left\|Y_{n}\right\|_{2}+\left\|Z_{n}\right\|_{2}\right)^{2} \leq 2\left(\left\|Y_{n}\right\|_{2}^{2}+\left\|Z_{n}\right\|_{2}^{2}\right) \text {. }
$$

From this fact and Lemma 3 we deduce that

$$
\begin{aligned}
& \sum_{n \geq 2}^{*}(n-1)(n+d-1)\left\|X_{n}\right\|_{2}^{2} \leq 2 \sum_{n \geq 2}^{*}(n-1)(n+d-1)\left\|Y_{n}\right\|_{2}^{2} \\
&+2 \sum_{n \geq 2}^{*}(n-1)(n+d-1)\left\|Z_{n}\right\|_{2}^{2} \\
& \leq 2 d(d-1)(\delta(K)+\delta(L)) .
\end{aligned}
$$

Combining (10), (11), and the fact that (9) implies $\left\|X_{0}\right\|_{2}^{2} \leq \varepsilon^{2} / 4$, we finally obtain

$$
h_{2}(K, L)^{2}=\sum^{*}\left\|X_{n}\right\|_{2}^{2} \leq\left(\frac{\varepsilon}{2}\right)^{4 / d}\left(\frac{2 d(d-1)^{2}}{\mu_{d}^{4 /(d-2)}}(\delta(K)+\delta(L))+\frac{\varepsilon^{2}}{4}\right)^{d(d-2) / d},
$$

which is the desired inequality.

\section{CONVEX Bodies OF CONSTANT WIDTH}

We now return to the problem of Minkowski that was discussed in the introduction. If $K$ is any convex body in $\mathbb{E}^{d}$, we let $K^{*}$ denote the convex body obtained from $K$ by central symmetrization, that is, $K^{*}=\frac{1}{2}(K+(-K))$. Furthermore, if $u \in S^{d-1}$ we let $w_{K}(u)$ denote the width of $K$ in the direction $u$. Hence, $w_{K}(u)=H_{K}(u)+H_{K}(-u)$. Two convex bodies $K, L$ in $\mathbb{E}^{d}$ are said to be equiwide if for all $u \in S^{d-1}$ we have $w_{K}(u)=w_{L}(u)$. Thus, convex bodies of constant width are exactly those which are equiwide to a ball. For convenience of notation we set

$$
\phi(\varepsilon)=\varepsilon^{2 / d} \frac{1}{2}\left(\lambda_{d}(\delta(K)+\delta(L))+\varepsilon^{2}\right)^{(d-2) / 2 d},
$$

with $\lambda_{d}$ as in Theorem 1 . We can then formulate the following extension of Minkowski's theorem. 
Theorem 2. Let $K$ and $L$ be two convex bodies in $\mathbb{E}^{d}$ and assume that for some $\varepsilon \geq 0$

$$
\left\|M\left(K_{u}\right)-M\left(L_{u}\right)\right\|_{2} \leq \varepsilon
$$

Then

$$
\left\|w_{K}(u)-w_{L}(u)\right\|_{2} \leq 2 \phi(\varepsilon) .
$$

Proof. Since $M\left(K_{u}\right)=M\left(K_{u}^{*}\right)$ and $M\left(L_{u}\right)=M\left(L_{u}^{*}\right)$, we have

$$
\left\|M\left(K_{u}^{*}\right)-M\left(L_{u}^{*}\right)\right\|_{2} \leq \varepsilon
$$

and so Theorem 1 yields

$$
\left\|w_{K}(u)-w_{L}(u)\right\|_{2}=\left\|w_{K^{*}}(u)-w_{L^{*}}(u)\right\|_{2}=2 h_{2}(K, L) \leq 2 \phi(\varepsilon) .
$$

In the special case when $L$ is a ball of diameter $c$ we obtain the following result.

Corollary 1. Let $K$ be a convex body in $\mathbb{E}^{d}$ and assume that for some $\varepsilon \geq 0$

$$
\left\|M\left(K_{u}\right)-c\right\|_{2} \leq \varepsilon .
$$

Then

$$
\left\|w_{K}(u)-c\right\|_{2} \leq \psi(\varepsilon),
$$

where $\psi(\varepsilon)=\varepsilon^{2 / d}\left(\lambda_{d} \delta(K)+\varepsilon^{2}\right)^{(d-2) / 2 d}$, with $\lambda_{d}$ as in Theorem 1 .

Here the problem arises of estimating the deviation of a convex body $K$ from a convex body of constant width if the width of $K$ is approximately constant. The following result is of interest in this connection.

Proposition 1. Let $K$ be a convex body in $\mathbb{E}^{d}$ with the property that for some $c \geq 0$ and $\eta \geq 0$

$$
\left|w_{K}(u)-c\right| \leq \eta \quad\left(\text { for all } u \in S^{d-1}\right) .
$$

Then there is a convex body $C$ of constant width $c$ such that

$$
h(K, C) \leq 3 \eta \text {. }
$$

Proof. Let $B_{t}$ denote the closed ball of diameter $t$ centered at the origin and let $C_{0}$ denote a completion of $K$. By this we mean a convex body that contains $K$ and is of constant width $d(K)$, the diameter of $K$; see Bonnesen and Fenchel [3] for details. Now (13) obviously implies

$$
|d(K)-c| \leq \eta \text {. }
$$

We may assume that $o \in C_{0}$ and then define $C=\frac{c}{d(K)} C_{0}$. We also note that for any pair of convex bodies $A, B$ in $\mathbb{E}^{d}$ with $A \subset B$ we have

$$
\begin{aligned}
h(A, B) & =\sup _{u}\left(H_{B}(u)-H_{A}(u)\right) \\
& \leq \sup _{u}\left(H_{B}(u)-H_{A}(u)+H_{B}(-u)-H_{A}(-u)\right) \\
& =2 h\left(A^{*}, B^{*}\right) .
\end{aligned}
$$


Using this remark, (13), and (14), we find

$$
\begin{aligned}
h(K, C) & \leq h\left(K, C_{0}\right)+h\left(C_{0}, C\right) \\
& \leq 2\left(h\left(K^{*}, C_{0}^{*}\right)+h\left(C_{0}^{*}, C^{*}\right)\right)=2\left(h\left(K^{*}, B_{d(K)}\right)+h\left(B_{d(K)}, B_{c}\right)\right) \\
& \leq 2\left(h\left(K^{*}, B_{c}\right)+2 h\left(B_{d(K)}, B_{c}\right)\right) \leq 3 \eta .
\end{aligned}
$$

Combining this proposition with Corollary 1 and (1), we obtain the following stability version of Minkowski's theorem (note that $\left\|w_{K}(u)-c\right\|_{2}=2 h_{2}\left(K^{*}, B_{c}\right.$ ) and $\left.\sup _{u}\left|w_{K}(u)-c\right|=2 h\left(K^{*}, B_{c}\right)\right)$.

Corollary 2. Let $K$ be a convex body in $\mathbb{E}^{d}$ and assume that for some $c \geq 0$ and $\varepsilon \geq 0$

$$
\left\|M\left(K_{u}\right)-c\right\|_{2} \leq \varepsilon .
$$

Then, there is a convex body $C$ of constant width $c$ such that

$$
h(K, C) \leq 4 \alpha_{2}\left(2 D^{2}\right)^{(d-1) /(d+1)} \psi(\varepsilon)^{2 /(d+1)},
$$

where $D=\max \{d(K), c\}, \psi$ is as in Corollary 1 , and $\alpha_{2}$ is as in (1).

\section{A STABILITY PROPERTY OF A GEOMETRIC INEQUALITY}

Although it is not part of the principal aim of the present investigation, we add a short discussion concerning a geometric inequality that is obtained as a by-product of the proof of Lemma 3. It concerns the expression

$$
\delta(\cdot)=\frac{1}{\kappa_{d}} W_{d-1}^{2}(\cdot)-W_{d}(\cdot)
$$

introduced in Theorem 1. It is one of the well-known inequalities of geometry that $\delta(K) \geq 0$ with equality occurring exactly when $K$ is a ball; see, for example, Aleksandrov [1] or Leichtweiss [10]. We consider the stability problem of estimating the deviation of $K$ from a ball if $\delta(K) \leq \varepsilon$. The following result addresses this question.

Theorem 3. Let $K$ be a convex body in $\mathbb{E}^{d}$ and let $B$ be the ball whose center is at the Steiner point of $K$ and whose radius is $\frac{1}{2} M(K)$. Then

$$
\frac{1}{\kappa_{d}} W_{d-1}^{2}(K)-W_{d-2}(K) \geq \frac{d+1}{d(d-1)} h_{2}(K, B)^{2} .
$$

Equality holds if and only if $K$ has a support function of the form $U_{0}+U_{1}+U_{2}$, where $U_{k}$ denotes a spherical harmonic of order $k$.

Proof. As in Lemma 3 we let $G$ be the support function of $K$ and assume $G \sim \sum_{n=0}^{\infty} U_{n}$. We may also assume that the Steiner point of $K$ is $o$. Then we have $U_{1}=0$ and $U_{0}=\frac{1}{2} M(K)$, and Lemma 3 shows that

$$
\begin{aligned}
d(d-1) \delta(K) & \geq \sum_{n=2}^{\infty}(n-1)(n+d-1)\left\|U_{n}\right\|_{2}^{2} \geq \sum_{n=2}^{\infty}(d+1)\left\|U_{n}\right\|_{2}^{2} \\
& =(d+1)\left\|\sum_{n=0}^{\infty} U_{n}-U_{0}\right\|_{2}^{2}=(d+1) h_{2}(K, B)^{2} .
\end{aligned}
$$


Since it is obvious that equality holds exactly if $U_{3}=U_{4}=\cdots=0$, the theorem is proved.

We conclude with several remarks regarding Theorem 3. A convex body $K$ is called a rotor in a polytope $P$ if for any rotation $\rho K$ of $K$ there is a translation vector $p_{\rho} \in \mathbb{E}^{d}$ such that $\rho K+p_{\rho} \subset P$ and $\rho K+p_{\rho}$ touches all facets of $P$. Schneider [16] has shown that in the case $d \geq 4$, a convex body is a rotor in a regular simplex if and only if its support function is of the form $U_{0}+U_{1}+U_{2}$, where $U_{n}$ is a spherical harmonic of order $n$. Thus if $d \geq 4$, Theorem 3 yields a characterization of rotors in a regular simplex in terms of an extremal property. (If $d=2,3$ convex bodies with a support function of the form $U_{0}+U_{1}+U_{2}$ are rotors in a regular simplex, but the converse is not true.)

It is surprising that in contrast to most inequalities of this kind, our inequality involves no limitation on the size or degeneracy of $K$.

Theorem 3 can be generalized to mixed volumes of the form $v(K, L)=$ $V(K, L, B, \ldots, B)$, where the unit ball $B$ appears $d-2$ times. Using this notation we can obtain the following result:

If $K$ and $L$ are convex bodies in $\mathbb{E}^{d}$, and $\bar{K}, \bar{L}$ are homothetic copies of $K$ and $L$ with $M(\bar{K})=M(\bar{L})=1$ and coincident Steiner points, then

$$
v(K, L)^{2}-v(K, K) v(L, L) \geq \frac{d+1}{d(d-1)} M(L)^{2} v(K, K) h_{2}(\bar{K}, \bar{L})^{2} .
$$

The proof of this inequality is similar to that of (15). Performing suitable homothetic transformations, we may assume $K=\bar{K}, L=\bar{L}$. Obviously,

$$
2 v(K, L)=v(K+L, K+L)-v(K, K)-v(L, L),
$$

and therefore

$$
\begin{aligned}
v(K, L)^{2}-v(K, K) v(L, L) \\
\geq \\
\quad(v(K, K)-v(K, L))^{2}-v(K, K) \\
\quad \times(v(K, K)-2 v(K, L)+v(L, L)) \\
\geq-v(K, K)(2 v(K, K)+2 v(L, L)-v(K+L, K+L)) .
\end{aligned}
$$

If $\sum_{n=0}^{\infty} X_{n}$ and $\sum_{n=0}^{\infty} Y_{n}$ are again spherical harmonic expansions of the support functions of $K$ and $L$, then $\sum_{n=0}^{\infty}\left(X_{n}+Y_{n}\right)$ is the corresponding expansion for $K+L$. Using the fact that $v(C, C)=W_{d-2}(C)$, and the representations (6) and (7) of $W_{d-2}$ in terms of spherical harmonics, we deduce from (17) that

$$
v(K, L)^{2}-v(K, K) v(L, L) \geq \frac{1}{d(d-1)} W_{d-2}(K) \sum_{n=0}^{\infty}(n-1)(n+d-1)\left\|X_{n}-Y_{n}\right\|^{2} .
$$

This obviously implies (16) in the case $K=\bar{K}, L=\bar{L}$, since in this case $X_{0}=Y_{0}=1$ and $X_{1}=Y_{1}$.

For a somewhat different approach to inequalities of this type, see Kubota [9], Schneider [17], and regarding the case $d=2$, Groemer [7]. 


\section{REFERENCES}

1. A. D. Aleksandrov, Zur Theorie der gemischten Volumina von konvexen Körpern II. Neue Ungleichungen Zwischen den gemischten Volumina und ihre Anwendungen, Mat. Sb. (N.S.) 2 (1937), 1205-1238. (Russian)

2. C. Berg, Corps convexes et potentiels sphériques, Danske Vid. Selsk. Mat-fys. Medd. 37 (1969), 1-64.

3. T. Bonnesen and W. Fenchel, Theorie der konvexen Körper, Chelsea, New York, 1977.

4. J. Bourgain and J. Lindenstrauss, Projection bodies, Geometric Aspects of Functional Analysis (J. Lindenstrauss and V. D. Milman, eds.) Lecture Notes in Math., vol. 1317, SpringerVerlag, New York, 1988, pp. 250-270.

5. S. Campi, Reconstructing a convex surface from certain measurements of its projection, Boll. Un. Mat. Ital. B 5 (1986), 945-959.

6. P. Goodey, Instability of projection bodies, Geom. Dedicata 20 (1986), 295-305.

7. H. Groemer, Stability properties of geometric inequalities, Amer. Math. Monthly (to appear).

8. S. Helgason, Groups and geometric analysis, Academic Press, Orlando, FL, 1984.

9. T. Kubota, Über die konvex-geschlossenen Mannigfaltigkeiten im n-dimensionalen Raum, Tôhoku Univ. Sci. Rep. 14 (1925), 85-88.

10. K. Leichtweiss, Konvexe Mengen, Springer, Berlin, 1980.

11. H. Minkowski, Über die Körper konstanter Breite, Teubner, Leipzig-Berlin, 1911, pp. 277279.

12. C. Müller, Spherical harmonics, Lecture Notes in Math., vol. 17, Springer, Berlin, 1966.

13. R. Schneider, Zu einem Problem von Shephard über die Projektionen konvexer Körper, Math. Z. 101 (1967), 71-82.

14. __ Functions on a sphere with vanishing integrals over certain subspheres, J. Math. Anal. Appl. 26 (1969), 381-384.

15. __, Functional equations connected with rotations and their geometric applications, Enseign. Math. (2) 6 (1970), 297-305.

16. __ Gleitkörper in konvexen Polytopen, J. Reine Angew. Math. 248 (1971), 193-220.

17. __ Stability in the Aleksandrov-Fenchel-Jessen theorem, Mathematika 36 (1989), 50-59.

18. R. Schneider and W. Weil, Zonoids and related topics, Convexity and its Applications (P. M. Gruber and J. M. Wills, eds.) Birkhäuser, Basel, 1983.

19. R. Vitale, $L_{p}$ metrics for compact convex sets, J. Approx. Theory 45 (1985), 280-287.

Mathematics Department, University of Oklahoma, Norman, Oklahoma 73072

Department of Mathematics, University of Arizona, Tucson, Arizona 85721 